\title{
Improving the confidence of "questioned versus known" fiber comparisons using microspectrophotometry and chemometrics
}

Georgina Sauzier $^{\mathrm{a}, \mathrm{b}}$, Eric Reichard ${ }^{\mathrm{c}}$, Wilhelm van Bronswijk ${ }^{\mathrm{a}}$, Simon W. Lewis ${ }^{\mathrm{a}, \mathrm{b}}$ and John V. Goodpaster ${ }^{\mathrm{c}, *}$

\author{
${ }^{a}$ Department of Chemistry, Curtin University, GPO Box U1987, Perth, Western Australia, 6845. \\ ${ }^{b}$ Nanochemistry Research Institute, Curtin University, GPO Box U1987, Perth, Western Australia, 6845. \\ c Department of Chemistry and Chemical Biology, Indiana University Purdue University Indianapolis (IUPUI), \\ Indianapolis, IN 46202. E-mail: jvgoodpa@iupui.edu.au; Tel: +1 3172746881
}

*Author for correspondence

\author{
Author e-mails: \\ Georgina Sauzier: Georgina.Sauzier@postgrad.curtin.edu.au \\ Eric Reichard: reicharde@yahoo.com \\ Wilhelm van Bronswijk: W.vanBronswijk@curtin.edu.au \\ Simon W. Lewis: S.Lewis@curtin.edu.au \\ John V. Goodpaster: jvgoodpa@iupui.edu
}

\begin{abstract}
Microspectrophotometry followed by chemometric data analysis was conducted on pairs of visually similar blue acrylic fibers, simulating the "questioned versus known” scenarios often encountered in forensic casework. The relative similarity or dissimilarity of each pair was determined by employing principal component analysis, discriminant analysis and Fisher's exact test. Comparison of fibers from within each set resulted in a correct inclusion result in 10 out of 11 scenarios, with the one false exclusion attributed to a lack of reproducibility in the spectra. Comparison of fibers from different sets resulted in a correct exclusion result in 108 of 110 scenarios, with two sets that shared identical dye combinations being indistinguishable. Although the presented methods are not infallible, they may nonetheless provide a path forward for forensic fiber examiners that has a more scientifically rigorous basis on which to support their findings in a court of law.
\end{abstract}

Keywords: Forensic science, textile fibers, microspectrophotometry, chemometrics 


\section{Introduction}

Textile fibers are a commonly encountered form of forensic trace evidence, and may be used to provide evidence of association due to their high tendency to shed and be transferred through physical contact [1-3]. Furthermore, certain classes of fibers can prove highly distinctive based on their morphology, composition and especially color. Over 7,000 textile dyes and pigments are currently produced worldwide, with combinations of these often used to impart specific colors to textile products $[2,4]$. Additionally, textile dyeing processes are generally carried out in batches that may exhibit minor variations in dye form, shade or strength [5]. Although many colors can be distinguished visually, these assessments are subjective and may be affected by metamerism or the examiner's color vision [6, 7]. More objective measurements can be obtained using instrumental methods such as microspectrophotometry (MSP), which is favored as a rapid and non-destructive method for characterizing the color of dyed fibers [8, 9]. Several studies have demonstrated the capability of MSP to distinguish visually similar colored fibers based upon different chromophores in the molecular structure of their dyes [10-14].

Forensic fiber examinations frequently involve comparisons between a questioned (Q) sample recovered from a crime scene and a known (K) sample taken from a known source such as a suspect's home or belongings [15]. Such comparisons may result in one of three outcomes: inclusion (e.g., "Q and K may have originated from the same source”), exclusion (e.g., "Q $\mathrm{Q}$ and K did not originate from the same source”), or an inconclusive result. In the case of textile fibers, which are mass produced, a "source” can only be described in terms of a class of objects that share the same physical and chemical characteristics rather than any particular item.

The underlying logic of these "questioned versus known” (Q vs. K) comparisons can be expressed through the following if/then statement:

"IF $\mathrm{Q}$ and $\mathrm{K}$ originated from the same source, THEN $\mathrm{Q}$ and $\mathrm{K}$ will share indistinguishable class characteristics.”

It follows, then, that the contrapositive must also be true:

"IF Q and K do not share indistinguishable class characteristics, THEN Q and K did not originate from the same source”. 
It is critical to realize, however, that it is a fallacy to affirm the consequent:

"IF Q and K share indistinguishable class characteristics THEN Q and K originated from the same source”.

This means, therefore, that any forensic comparison that results in an association based upon indistinguishable characteristics may be a false inclusion. There is also an implicit assumption for any given Q vs. K comparison that the source(s) of Q and K are homogenous, i.e. that both the questioned and known are representative samples of their original source(s). If this assumption is invalid, it may lead to false exclusions (see contingency table below).

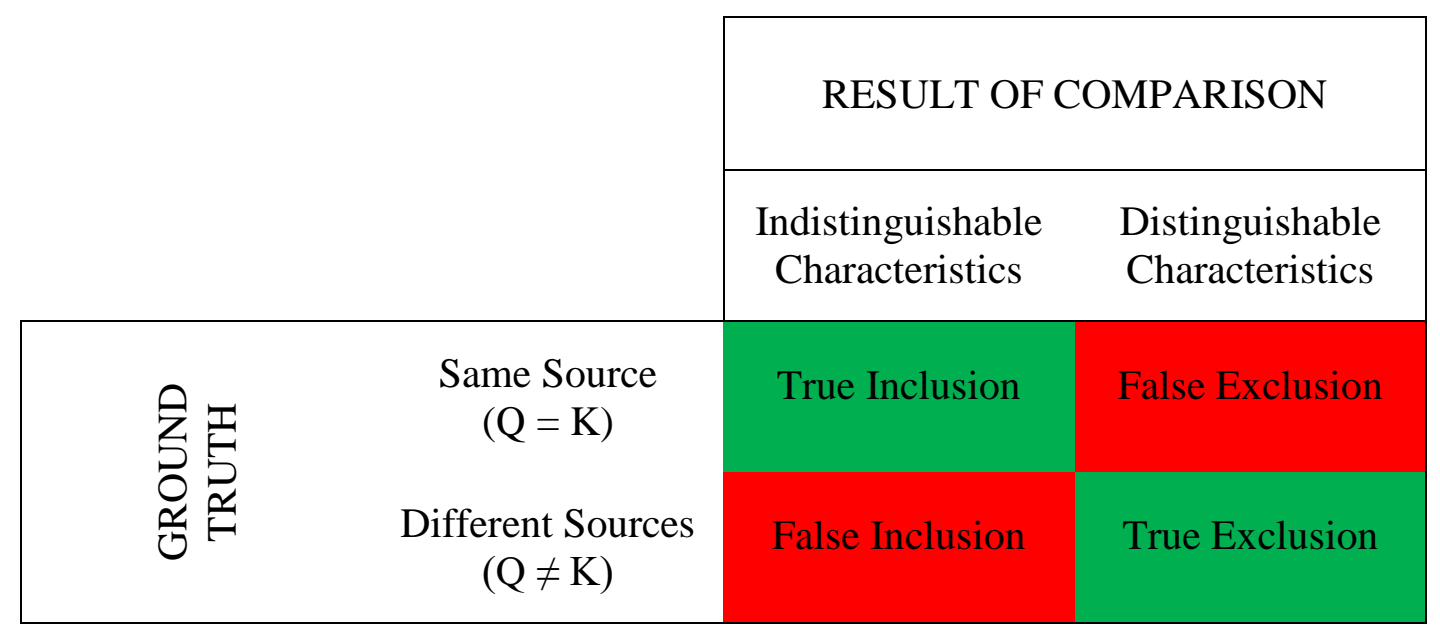

In general, the relative strength of an inclusion depends upon the rarity of the class to which Q and $\mathrm{K}$ are assigned, as the chance of a coincidental inclusion goes down as the source of $\mathrm{Q}$ and $\mathrm{K}$ becomes smaller and less common. A more quantitative approach to describing these outcomes can be expressed by stating two competing hypotheses:

Prosecutor's Hypothesis $\left(\mathbf{H}_{\mathbf{p}}\right)$ : The questioned fiber(s) originate from the individual/object which is the source of the known. This hypothesis represents a "true inclusion”.

Defense Hypothesis (Hd): The questioned fiber(s) originate from another individual/object than the one suspected. This hypothesis represents a "false inclusion". 
In turn, a likelihood ratio (LR), as derived from the Bayes Theorem can be defined as:

$\mathrm{LR}=\mathrm{P}\left(\mathrm{E} \mid \mathrm{H}_{\mathrm{p}}\right) / \mathrm{P}\left(\mathrm{E} \mid \mathrm{H}_{\mathrm{d}}\right)$

Where evidence (E) can be a quantitative score of similarity or dissimilarity between the questioned and known, $\mathrm{P}\left(\mathrm{E} \mid \mathrm{H}_{\mathrm{p}}\right)$ is the probability of observing the evidence given the prosecutor's hypothesis and $\mathrm{P}\left(\mathrm{E} \mid \mathrm{H}_{\mathrm{d}}\right)$ is the probability of observing the evidence given the defense hypothesis.

Ultimately, a fiber comparison that utilizes an analytical method such as MSP includes a determination of whether the spectra from a Q and K are truly "indistinguishable”. Such a determination depends upon the variation between spectra of the questioned and known samples. Specifically, guidelines published by the Scientific Working Group for Materials Analysis (SWGMAT) dictate that a 'spectral inclusion' can be made if the questioned spectrum lies within the range of the known spectra in terms of the curve shape and absorbance values [16].

Traditionally, assessment of whether two or more fibers exhibit similar spectral characteristics has relied upon an examiner's visual interpretation of the data. The subjective nature of these comparisons has led to trepidations regarding potential human error or bias [17]. Substantial research in recent decades has therefore examined the utility of analytical techniques with chemometric analysis to provide more objective fiber examinations [18-22]. Liu for example employed Raman spectroscopy with chemometrics to distinguish cotton cellulose fibers based upon their color, crystalline fraction and strength [23]. Morgan et al. also described several inter-laboratory studies employing chemometrics with UV-vis and fluorescence MSP to a large database of dyed fibers, discriminating fibers according to both their dye composition and loadings with high levels of accuracy [24]. However, these studies have largely focused on the simultaneous discrimination of several fibers, rather than the Q vs. K comparisons more typical of forensic casework. Furthermore, there is presently a lack of quantitative measures for assessing sample similarity. The establishment of cut-off criteria for an 'inclusion' or ‘exclusion' result would provide an additional statistical basis on which forensic practitioners could support their findings in a court of law. 
This study investigated the potential use of MSP spectroscopy followed by chemometrics to assess the similarity or dissimilarity of several blue-dyed acrylic fiber sets. Chemometric data analysis was conducted on spectra acquired from various fiber pairs in order to simulate casework Q vs. K comparisons. Quantitative determination of the similarity was then made by comparing the resultant data using hypothesis testing.

\section{Materials and methods}

\section{$2.1 \quad$ Samples}

Fiber samples were provided by the University of South Carolina. The sample population consisted of eleven sets of bilobal blue acrylic fibers colored with varying combinations of cationic (basic) dyes, as shown in Table 1. Representative images of each fiber set are provided in the electronic supplementary information (Figure S1). Fibers from each set had varying diameters as indicated.

Table 1: Dye compositions of eleven blue acrylic fiber sets utilized in this study.

\begin{tabular}{lll}
\hline Fiber Set & Dye composition & Diameter $(\boldsymbol{\mu m})$ \\
\hline Fiber A & Blue 3, Red 18, Yellow 28 & 17.5 \\
Fiber B & Blue 41, Red 46, Yellow 28, Yellow 29 & 15 \\
Fiber C & Blue 41, Red 46, Yellow 28 & 15 \\
Fiber D & Blue 41, Red 29, Yellow 21 & 21.25 \\
Fiber E & Blue 147, Red 29, Yellow 28 & 23.75 \\
Fiber F & Blue 3, Blue 147 & 23.75 \\
Fiber G & Blue 147, Red 46, Yellow 28 & 18.75 \\
Fiber H & Blue 3, Red 18, Yellow 28 & 22.5 \\
Fiber I & Blue 41, Red 29, Yellow 28 & 22.5 \\
Fiber J & Blue 41, Red 18, Yellow 28 & 25 \\
Fiber K & Blue 3, Red 46, Yellow 28 & 25 \\
\hline
\end{tabular}

\subsection{Microspectrophotometry}

Individual fibers from each set were randomly removed and mounted on glass microscope slides using Permount mounting media (Fisher Scientific, U.S.A) for analysis. MSP spectra were acquired between 400-800 nm using a CRAIC QDI 2000 microspectrophotometer in transmission mode, operated at 150x magnification. The spectrometer was calibrated using NIST traceable standards prior to use. An autoset optimization, dark scan and reference scan 
were also obtained prior to each sample analysis. Ten fibers were analyzed from each set, with five spectra recorded along the length of each to account for intra-fiber variation. Fifty averaged scans at a resolution of $5 \mathrm{~nm}$ were obtained for each spectrum.

\subsection{Data analysis}

Data pre-processing and chemometric analysis was conducted using XLSTAT (AddInSoft, Paris, France) and Unscrambler X 10.3 (Camo Software AS, Oslo, Norway). All spectra were first baseline offset to $0 \%$ absorbance and normalized to account for variations associated with the fiber diameter. In this case normalization to "unit vector length" was chosen as it was appropriate for UV-vis spectra [25]. Other normalizations were explored (i.e., normalization to unit area) but the performance of the model was not improved. Principal component analysis (PCA) was then conducted on the entire dataset of known sample spectra using Unscrambler X 10.3 .

The Q vs. K approach was undertaken by conducting PCA on pairs of fiber sets using XLSTAT. In each comparison, the "known” sample was defined as a group of 45 spectra originating from the first nine fibers of the set, and the "questioned" sample was defined as the five spectra acquired from the last fiber analyzed in the same set, or the last fiber analyzed in a different set. Discriminant analysis (DA) was performed in XLSTAT on each pair based on their PCA scores against the first three PCs (accounting for $>98 \%$ of total variance in each comparison), calculating prior membership probabilities from each training set. The number of PCs used to construct each model was selected according to the corresponding scree plots. All DA comparisons employed the Mahalanobis distance measure and assumed non-equal covariance matrices. This approach was repeated for each possible pair of questioned and known samples, yielding a total of 121 comparisons amongst all eleven fiber sets. Sample similarity was then evaluated using the classification results of the questioned spectra, mean membership probabilities and Fisher's exact test.

\section{Theory}

\subsection{Chemometric methods}

Analytical methods such as microspectrophotometry generate a large quantity of multivariate data, posing difficulties in objective interpretation. This issue can potentially be overcome through the use of multivariate statistical (chemometric) methods, such as PCA [26-28]. PCA 
reduces the dimensionality of complex data by transforming the original set of correlated variables into a lesser number of new, orthogonal variables referred to as principal components (PCs) [29-31]. Each successive PC is calculated to describe the maximum proportion of variation in the dataset that is not described by the previous components, such that the majority of information is retained within the first few PCs. These PCs may then be used as a new coordinate system to re-visualize the dataset, revealing patterns or relationships between samples that would not be readily evident from the raw data alone [32, 33]. Further information can be gleaned through inspection of the loadings plots, which indicate the variables of the original dataset that significantly contribute to the separation of samples along each PC [34].

Following the identification of sample groupings within a dataset, it is often of interest to develop classification models for future samples. This can be done using discriminant analysis (DA); a technique that builds a mathematical function to maximize the separation between known classes of samples [35, 36]. The subsequent model can then be employed to assign unknown samples to the most probable class. As the sample size of each group must exceed the number of variables, DA is typically carried out following data reduction techniques such as PCA, with the first few PCs being substituted in place of the original variable set $[37,38]$.

\subsection{Sample similarity metrics}

Due to the large size difference between the questioned and known classes, the overall classification accuracy of the discriminant model could not be taken as a reliable measure of differentiation. As only five of the 50 spectra involved in each comparison originated from the questioned sample, an overall classification accuracy of $90 \%$ would be obtained even if all questioned spectra were assigned to the known class. Similarly, the overall performance of the discriminant function as expressed by the area under a receiver operator characteristic curve could still be near unity, despite poor classification of the questioned spectra. The differentiation between each pair was therefore evaluated according to the percentage of questioned spectra assigned to the known class, assessing the extent to which the questioned sample fell within the boundaries of the known source (as recommended by the current SWGMAT guidelines [16, 39]). In addition, the mean membership probability of the questioned sample belonging to the known class was recorded.

Fisher's exact test was then used to obtain a quantitative measure of sample similarity. This test is useful in the analysis of contingency tables resulting from the binary classification of 
objects, such as the classification of spectra as belonging to a questioned or known class. Fisher's test calculates the exact probability of observing a given combination of data in a contingency table, as described below [40, 41]. The null hypothesis ( $p>0.05$ at the $95 \%$ confidence level) is that the rows and columns of the table are independent, i.e., there is no association between the assignation of spectra to the questioned or known fibers and the actual source of the spectra [42]. This would indicate that the questioned and known fibers possess overlapping characteristics, and thus could potentially originate from a common source. Conversely, the alternative hypothesis $(\mathrm{p}<0.05)$ is that there is an association between the actual and predicted fiber sources. This in turn implies that the questioned and known fibers are distinguishable, and that they are likely to originate from different sources.

\section{Calculation}

\subsection{Fisher's test statistic}

Contingency tables were constructed from the results of the discriminant analysis conducted on each Q vs. K pair, as shown in Table 2.

Table 2: General form of a contingency table generated from a Q vs. K comparison.

\begin{tabular}{llll}
\hline & $\begin{array}{l}\text { Classified as } \\
\text { “Known” }\end{array}$ & $\begin{array}{l}\text { Classified as } \\
\text { “Questioned” }\end{array}$ & Total \\
\hline Known & $a$ & $b$ & $a+b$ \\
Questioned & $c$ & $d$ & $c+d$ \\
Total & $a+c$ & $b+d$ & \\
\hline
\end{tabular}

Fisher's test statistics were calculated from each contingency table according to the following formula, where $N$ is the sum of $a, b, c$, and $d$.

$$
p=\frac{(\mathrm{a}+\mathrm{b}) !(\mathrm{c}+\mathrm{d}) !(\mathrm{a}+\mathrm{c}) !(\mathrm{b}+\mathrm{d}) !}{\mathrm{a} ! \mathrm{b} ! \mathrm{c} ! \mathrm{d} ! \mathrm{N} !}
$$




\section{Results and discussion}

\subsection{Preliminary considerations}

Single textile fibers often exhibit varying levels of dye uptake, particularly where a multicomponent dye has been used [5, 6]. This potential heterogeneity both among and within individual fibers requires appropriate sampling to obtain representative data for the bulk material. For synthetic textiles such as acrylics, SWGMAT guidelines recommend the examination of at least five individual fibers, with a minimum of five replicate spectra acquired for each $[15,16]$. In this study, nine to ten fibers were hence utilized in each known set to ensure representative sampling. As it is not always possible in casework scenarios to recover multiple fibers from the questioned source, single fibers were chosen to act as the questioned sample. This was intended to simulate a challenging scenario wherein a single fiber is recovered and submitted for analysis alongside a much larger known source, such as a garment or blanket. However, the large difference in size of the questioned and known classes has the potential to affect the results obtained in this study, and this must be considered when evaluating the results.

MSP spectra from fiber sets A and H (Table 1) were each collected over two consecutive days, with the instrument re-calibrated on each date. In a casework scenario, the known and questioned fibers would ideally be analyzed on the same day, thus minimizing the risk of daily variations in the instrument performance affecting the results. However, this is not always feasible where a large number of samples have been submitted for analysis. The re-calibration of the instrument, in addition to inherent instrumental variability, may therefore result in spectral deviations that could influence the results determined through statistical analysis.

\subsection{Correlation between fiber spectra and component dyes}

Chemical structures of the component dyes used in this research are provided in the supplementary information (Figure S2). Although reference standards for each dye were not available, visual inspection of the spectra allowed broad correlations to be drawn between these dyes and specific spectral features (Table 3). 
Table 3: Spectral features attributable to dyes contained in the blue acrylic fibers.

\begin{tabular}{ll}
\hline Dye & Absorbance peak/s \\
\hline Blue 3 & Sharp peak at $655 \mathrm{~nm}$, shoulder at $600 \mathrm{~nm}$ \\
Blue 41 & Peak at $625 \mathrm{~nm}$, shoulder at $585 \mathrm{~nm}$ \\
Blue 147 & Broad peak at $600 \mathrm{~nm}$ \\
Red 46 & Peak at $545 \mathrm{~nm}$ \\
Yellow 21 & Peak at $450 \mathrm{~nm}$, possible peak at $425 \mathrm{~nm}$ \\
Yellow 28 & Peak at $450 \mathrm{~nm}$ \\
Yellow 29 & Peak at $450 \mathrm{~nm}$ \\
\hline
\end{tabular}

For the purposes of this inspection, the fiber sets were divided into three groups according to their primary blue dye: Blue 3 (sets A, F, H and K), Blue 41 (B, C, D, I and J) or Blue 147 (sets E and G). Example spectra from each of these fiber sets are shown in Figure 1. It was noted that spectra from sets $\mathrm{A}$ and $\mathrm{H}$ appeared visually identical, as expected given their identical dye combination (Table 1).

Fibers containing Blue 3 exhibited a strong peak at $655 \mathrm{~nm}$ with a shoulder at $600 \mathrm{~nm}$, consistent with known absorbance bands for this dye [43-45]. Sets A, H and K also showed minor peaks or shoulders at $450 \mathrm{~nm}$ consistent with Yellow 28 [46, 47]. No such peak was noted in the spectrum of set $\mathrm{F}$, as these fibers did not contain any yellow dyes. Set K spectra exhibited a weak shoulder at $545 \mathrm{~nm}$, possibly due to the presence of Red 46. Red 18, present in fibers from sets $\mathrm{A}$ and $\mathrm{H}$, gave no distinguishable peak in the corresponding spectra. Given the broad, overlapping nature of MSP spectra, it is possible that any absorbance band from this dye was masked by the blue or yellow dyes, which would likely have been present at much greater concentrations.

Samples containing Blue 41 showed a corresponding peak at $625 \mathrm{~nm}$, with a shoulder at 585 $\mathrm{nm}$. It is likely that the latter resulted in the masking of any red dyes, as none of the spectra yielded observable peaks in the red (500-550 nm) region. Fibers containing Yellow 28 gave the expected absorbance band at $450 \mathrm{~nm}$, with the exception of sets I and J, which instead exhibited broad shoulders at ca. $490 \mathrm{~nm}$. It is possible that this band shift is due to the overlap or interaction between multiple dyes contained in these samples. Fiber set B (containing Yellows 28 and 29) gave a single peak in the yellow region indistinguishable from that arising 
solely from Yellow 28, indicating that these dyes give rise to overlapping bands centred around $450 \mathrm{~nm}$. Set D gave a weak shoulder at ca. 425, consistent with Yellow $21[47,48]$.

Both fiber sets containing Blue 147 produced a single broad band at ca. 600 nm. Any Blue 147 contained in set F could was hence likely masked by the $600 \mathrm{~nm}$ shoulder of Blue 3, also explaining why this shoulder exhibited a broader peak width and greater intensity relative to the $655 \mathrm{~nm}$ peak compared to fibers containing only Blue 3. The Blue 147 peak may also be responsible for masking any bands arising in the red region due to Red 29 (set E) or Red 46 (set $G$ ). Set $G$ fibers gave no distinguishable peak in the yellow region, despite containing Yellow 28, indicating that the concentration of yellow dye in these fibers was too low to be detected under the conditions in this study. 

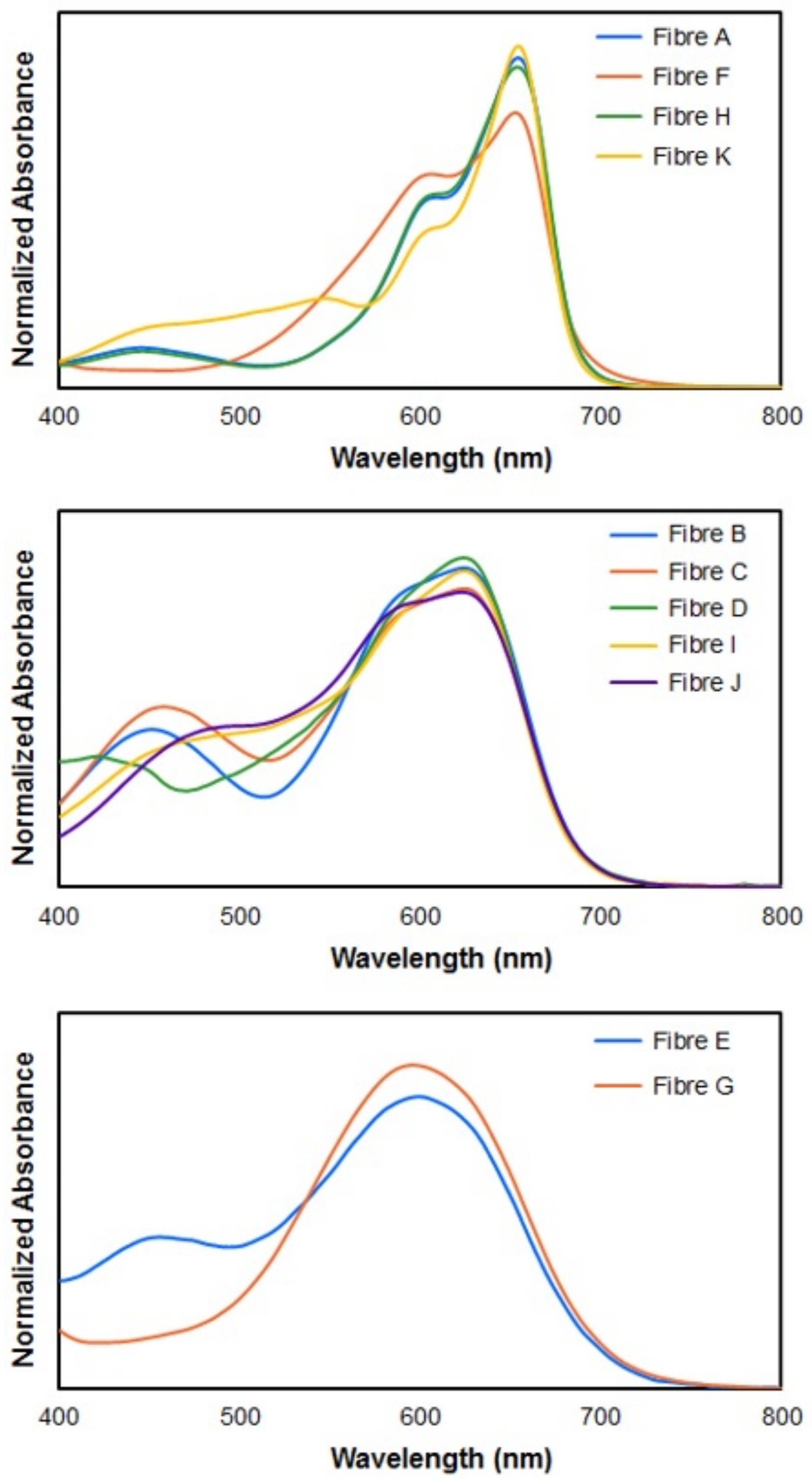

Figure 1: Normalized MSP spectra (each averaged across five replicates) for single blue acrylic fibers containing (top) Blue 3; (middle) Blue 41; and (bottom) Blue 147 as their primary blue dye. 


\subsection{Distribution of the spectral dataset}

PCA revealed that $96.6 \%$ of total variance in the dataset could be described by the first three PCs, as illustrated in the scree plot below (Figure 2). The scree plot is important in determining the optimum number of PCs to be retained within the model [29]. Retaining an insufficient number of PCs may cause information pertaining to dataset variation being lost, whilst extraneous PCs may result in the modelling of random variance or noise [29, 49]. By assessing the variance accounted for by each individual PC, and determining the point at which the curve begins to plateau, the optimum number of PCs required to model the data can be identified [32, 50]. In this case, the scree plot indicated that the use of up to four PCs (accounting for $98.5 \%$ of the total variation) was suitable to re-visualize the dataset.

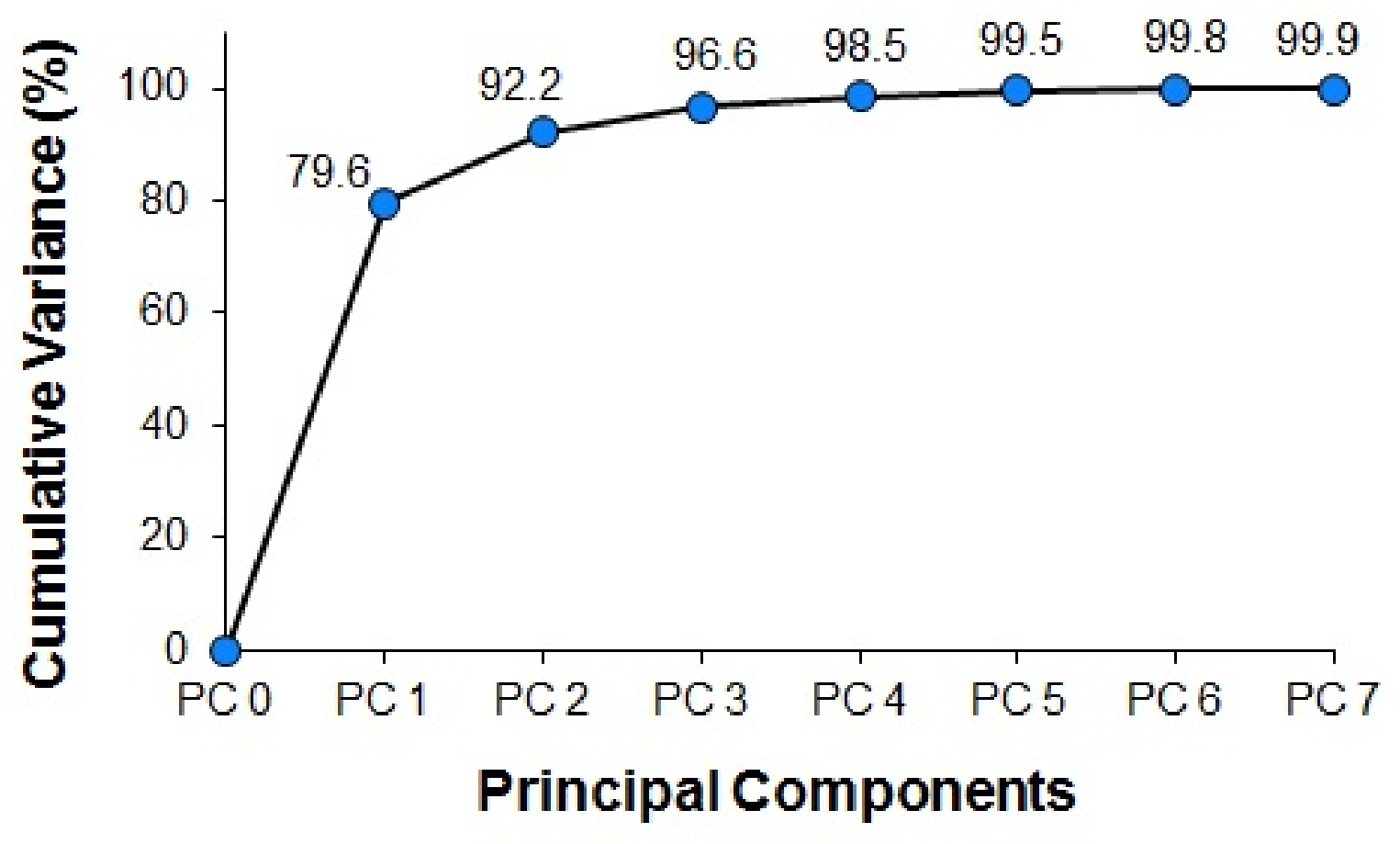

Figure 2: Scree plot depicting the cumulative variance in the dataset retained by each PC.

Scores plots generated using combinations of the first four PCs resulted in most fiber sets forming visually distinct clusters, with no obvious outliers (Figure 3). PC4, despite accounting for only $1.9 \%$ of variance within the dataset, was found to improve the discrimination between fiber sets $C$ and $D$. These exemplars were observed to overlap when utilizing only the first three PCs. Fiber sets A and H exhibited significant overlap, which was attributed to these fibers possessing the same dye combination (Table 1). It should also be noted, though, that these fibers have different diameters, and would hence be distinguishable based upon a general microscopic examination. 
Spectra from sets D and E exhibited a high level of spread (i.e. intra-class variance) compared to the remaining samples when employing the first three PCs. Visual inspection of the corresponding spectra (Figure S3) revealed variation in the relative absorbance between bands in the 400-500 nm (yellow dye) region and those in the $600 \mathrm{~nm}$ (blue dye) region. This is potentially due to differing dye uptake amongst individual fibers, as discussed above. This also reinforces the importance of collecting an adequate number of fibers and replicate spectra to allow representative measurements to be obtained.

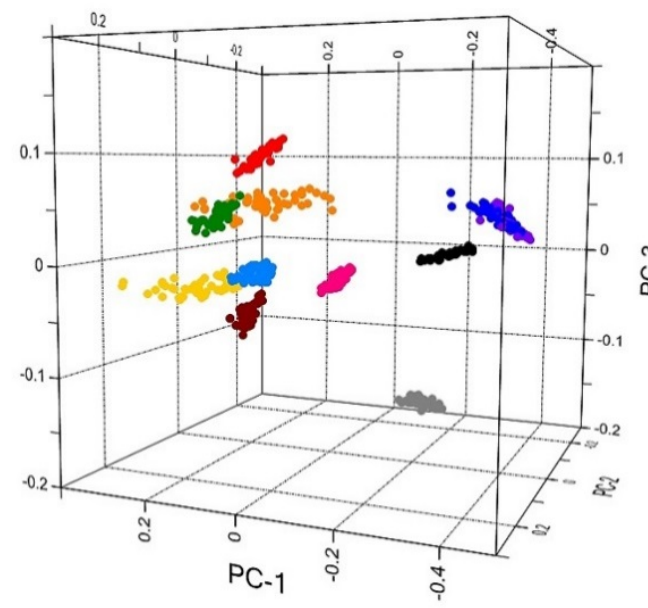

- Fibre Set A

- Fibre Set B

- Fibre Set C

- Fibre Set D

- Fibre Set E

- Fibre Set F

- Fibre Set G

- Fibre Set H

- Fibre Set I

- Fibre Set J

- Fibre Set K

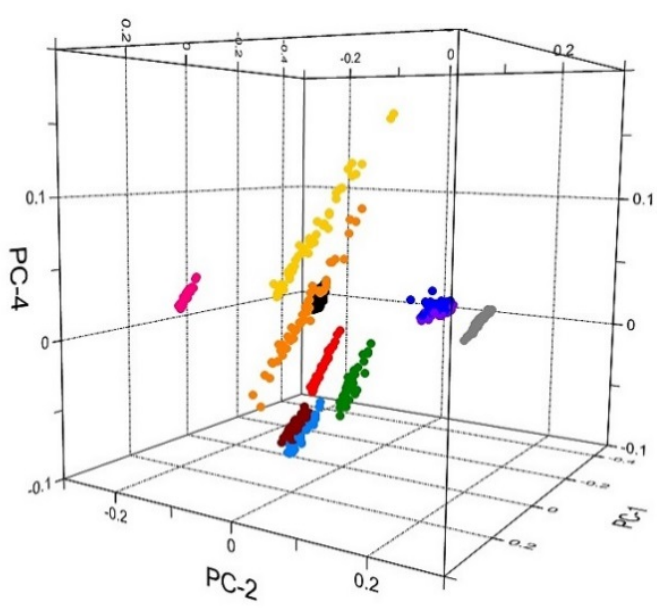

Figure 3: 3-dimensional PCA scores plot (employing PCs 1,2,3 and PCs 1,2,4) showing the distribution of blue acrylic fibers based upon their corresponding MSP spectra. Left and right images show the improved separation of Fiber Sets C and D upon the inclusion of PC4.

The factor loadings for the first four PCs (Figure 4) can be used to identify which wavelength regions are the largest contributors to variation between the fiber sets, and consequently between each Q vs. K pair. PC1 has a strong positive correlation at ca. 655 nm, consistent with the absorbance band for Blue 3 dye. Samples separated along this PC may therefore be assumed to differ in their relative proportions of this dye. For example, fibers from set $\mathrm{F}$ (containing Blue 3) attain positive scores against PC1, while fibers from set E (instead containing Blue 147) exhibit negative scores. Similarly, PC2 has a positive correlation at $450 \mathrm{~nm}$, consistent with Yellow 28 dye. Fiber pairs separated along this component are hence assumed to be dissimilar in terms of the type or concentration of yellow dye present. 


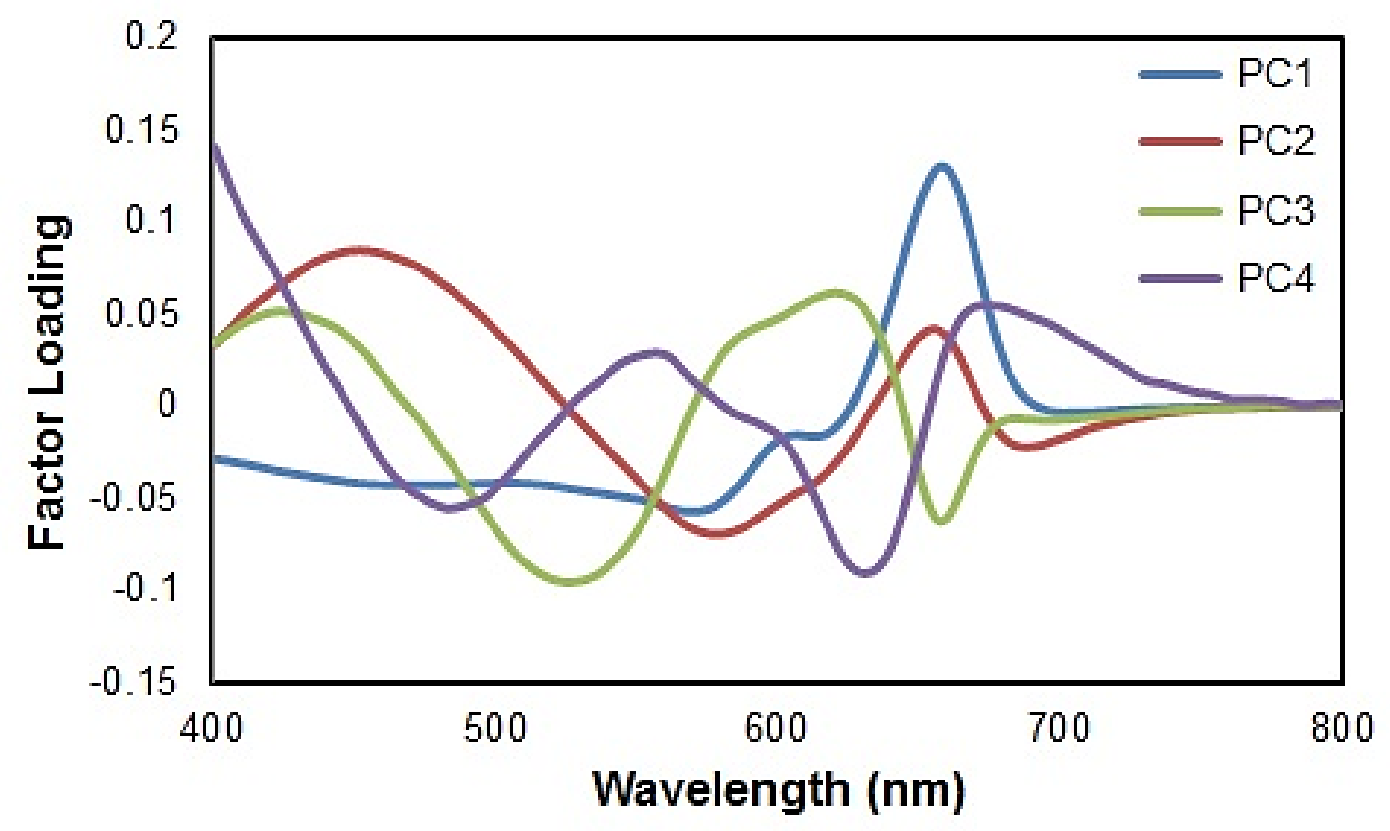

Figure 4: Factor loadings plot of PCs 1-4 for PCA of the entire blue acrylic dataset.

PC3 exhibits a strong negative correlation at approximately $530 \mathrm{~nm}$, consistent with a local minimum in the set B spectra (Figure 1). Set B fibers hence obtain the most positive scores along this PC, resulting in their separation from sets C, D, I or J. Likewise, PC4 has a negative correlation at ca. $470 \mathrm{~nm}$. At this wavelength, set D spectra exhibit a local minimum whilst set C spectra are near a maximum, resulting in the separation of these samples along PC4 as observed in the scores plot. PCA is thus not only valuable in qualitatively assessing the similarity of a questioned and known pair, but may reveal the chemical basis for their similarity or dissimilarity. Such relationships may be difficult to identify from visual examination of spectra, highlighting the potential utility of chemometrics in forensic applications.

\subsection{Simulated “ $Q$ vs. $K$ ” comparisons}

\subsubsection{Fibers from same source}

Simulated Q vs. K comparisons were undertaken by subjecting pairs of fiber sets to PCA and DA. Successful discrimination (100\% correct classification) was interpreted as an exclusion result wherein the fibers could be considered as distinguishable. Poor discrimination, wherein three or more of the questioned spectra were assigned to the known class, was treated as an inclusion - that is, the fibers could possibly originate from a common source. The first set of comparisons were carried out between fibers of the same set, employing the last fiber as the 
questioned sample and the remaining nine fibers as the known sample. These spectra were expected to be non-differentiable, thus yielding an inclusion result.

This was the case for ten of the eleven fiber sets, as shown in Table 4. The majority of the questioned spectra from these fiber sets were assigned to the known class, suggesting the questioned fiber to fall within the boundaries of the known source. Furthermore, the mean membership probability of these spectra belonging to the known class exceeded $50 \%$. The results of the Fisher Exact test largely agreed with these initial metrics, with large $p$-values obtained for nine of the fiber sets. Fiber set I generated a $p$-value just below 0.05 , which could be interpreted as an inconclusive comparison for this fiber set. For fiber set K, only two of the five questioned spectra were classified to the known class, and the mean membership probability for these two samples was less than $50 \%$. The Fisher test also resulted in a rejection of the null hypothesis, giving a false exclusion. This is potentially a result of fiber heterogeneity associated with dye concentration, as previously discussed.

Overall, these results indicate that while ten of eleven "same source" comparisons gave the expected result, there is a potential for false exclusions. This may be exacerbated by the large difference in size of the questioned and known classes, despite the fact that the initial probabilities of the discriminant model took this into account.

Table 4: Statistical values obtained from Q vs. K comparisons conducted on fibers from the same set. ${ }^{*}$ ) denotes that the spectra for the ten fibers in these fiber sets were each acquired over multiple consecutive days

\begin{tabular}{|c|c|c|c|}
\hline & $\mathbf{Q}=\mathbf{K}$ classification $(\%)$ & $\begin{array}{l}\text { Mean } \quad \text { Membership } \\
\text { Probability }(\mathrm{Q}=\mathrm{K})(\%)\end{array}$ & $\begin{array}{l}\text { Fisher's Exact Test } \\
\text { (p-value) }\end{array}$ \\
\hline Fiber Set $A^{*}$ & 100 & 76 & 1.0 \\
\hline Fiber Set B & 100 & 89 & 1.0 \\
\hline Fiber Set C & 100 & 87 & 1.0 \\
\hline Fiber Set D & 100 & 80 & 0.18 \\
\hline Fiber Set E & 100 & 88 & 1.0 \\
\hline Fiber Set F & 100 & 78 & 1.0 \\
\hline Fiber Set G & 100 & 74 & 1.0 \\
\hline Fiber Set $\mathrm{H}^{*}$ & 80 & 78 & 1.0 \\
\hline Fiber Set I & 60 & 53 & 0.04 \\
\hline Fiber Set J & 80 & 66 & 1.0 \\
\hline Fiber Set K & 40 & 42 & $5.1 \times 10^{-4}$ \\
\hline
\end{tabular}




\subsubsection{Fibers from different sources}

When comparing fibers originating from different sets, the majority of samples (with exception of sets A vs. $\mathrm{H}$ and $\mathrm{C}$ vs. D) were unambiguously differentiated, yielding true exclusions in 108 of 110 comparisons. In these comparisons, $100 \%$ correct classification was achieved and the membership probability of the questioned spectra belonging to the known class was determined to be $0 \%$. Successful discrimination of these fiber sets was expected based on their clear visual separation in the PCA scores plot. As these samples could be readily differentiated according to their PC scores, Fisher's test was deemed unnecessary and is not included here.

When fiber sets $\mathrm{C}$ and $\mathrm{D}$ were compared, there was a small probability for some of the questioned samples to be assigned to the known class (Table 5). This is consistent with the minor overlap noted between these sample sets in the PCA scores plot when employing the first three PCs. Nonetheless, as the probability associated with misclassification of the questioned spectra was minimal (below $5 \%$ in each case), the samples were still considered to be separable. Fisher's test also indicated that the Q vs. K membership frequencies for these samples were interdependent, yielding an overall exclusion result.

Table 5: Statistical values obtained from Q vs. K comparisons on fibers from different sets.

\begin{tabular}{|c|c|c|c|c|}
\hline \multirow[b]{2}{*}{ Questioned } & \multirow[b]{2}{*}{ Known } & \multirow[b]{2}{*}{$\begin{array}{l}Q \quad K \\
\text { classification (\%) }\end{array}$} & \multicolumn{2}{|l|}{ Mean } \\
\hline & & & $\begin{array}{l}\text { Membership } \\
\text { Probability } \\
(\mathrm{Q}=\mathrm{K})(\%)\end{array}$ & $\begin{array}{l}\text { Fisher's Exact } \\
\text { Test (p-value) }\end{array}$ \\
\hline Fiber Set C & Fiber Set D & 0 & 3 & $4.7 \times 10^{-7}$ \\
\hline Fiber Set D & Fiber Set C & 0 & 2 & $2.8 \times 10^{-6}$ \\
\hline Fiber Set A & Fiber Set H & 60 & 47 & $8.2 \times 10^{-3}$ \\
\hline Fiber Set H & Fiber Set A & 100 & 81 & 1.0 \\
\hline
\end{tabular}

When comparing fiber sets $\mathrm{A}$ and $\mathrm{H}$, the results were less conclusive. When a fiber from set $\mathrm{A}$ made up the questioned sample, the majority (60\%) of these spectra were assigned to the known class, although the mean membership probability was less than $50 \%$. Furthermore, Fisher's Exact test indicated that the two samples were distinguishable $(\mathrm{p}<0.001)$. However, when a fiber from set $\mathrm{H}$ made up the questioned sample, all questioned spectra were assigned to the known class with high probability and Fisher's Exact Test indicated that the two samples were indistinguishable. Taken together, this indicates that fiber sets $\mathrm{A}$ and $\mathrm{H}$ were not able to be reliably differentiated. 
The inability to discriminate between these sets was expected given their high degree of overlap in the PCA scores plot (Figure 3) and shared dye combination, although the relative concentrations of each dye component were not determined. Additionally, though the diameters of the fibers differed, normalization of the spectra was expected to nullify this difference. Interestingly, visual examination of the spectra from sets $\mathrm{A}$ and $\mathrm{H}$ revealed varying ratios of absorbance at the $655 \mathrm{~nm}$ peak and $650 \mathrm{~nm}$ shoulder (Figure S4). Both of these bands have been attributed to the same dye (Blue 3), and so heterogeneous dye uptake would not appear to be a contributing factor to this variation. As the spectra of these fiber sets were collected over consecutive days, it is likely that these deviations are a result of systematic instrumental variation or re-calibration, as discussed in section 5.1. For future studies, it would thus be more appropriate to collect data for these fiber sets in a single session, rather than analyzing the fiber sets sequentially over a number of days. Additionally, it should be noted in casework examinations where data has been acquired over multiple days, as this may similarly affect fiber examinations based upon visual data inspections.

\section{Conclusions}

The use of statistical methods with MSP shows great potential for rapidly distinguishing visually similar fibers on the basis of their dye combinations. The comparison of simulated questioned and known fibers from the same source resulted in a correct inclusion result for nine of the eleven fiber sets, although a false exclusion was obtained for fiber set $\mathrm{K}$, and fiber set I gave potentially ambiguous results. The comparison of questioned and known fibers from different sources allowed for the differentiation of all fiber sets with the exception of fiber set A and $\mathrm{H}$, which shared the same dye combination, yielding true exclusion results in 108 of 110 comparisons.

The single false exclusion was attributed to a lack of reproducibility in obtaining the MSP spectra. Spectra from several fiber sets (A, D, E and H) were also found to exhibit varying absorbance ratios between particular peaks. This may result from the heterogeneous uptake of multi-component dye mixtures by individual fibers. Alternatively, the analysis of samples across different dates could result in spectral deviations due to instrumental variability or the re-calibration of the instrument. Given the impact of these factors on the results obtained through statistical analyses, it can be concluded that these methods are not infallible. 
Nevertheless, the use of well-documented statistical protocols for these comparisons provides a more scientifically rigorous basis on which examiners can support their findings in court.

As this study employed a limited sample range, additional work is required to determine whether similar results can be obtained with other fiber or dye types. In particular, natural fibers such as cotton or wool are of interest due to their common use in modern textiles. The greater amount of natural variation amongst these fibers will also provide a more rigorous test of the protocols employed in this study. Finally, it should be noted that the exemplars utilized in this research were relatively new, and do not take into account potential effects due to laundering, every day wear, or similar factors which real samples may be subjected to. Studies incorporating such datasets will therefore be imperative for model validation, and to assess whether such models may be reliably applied to real scenarios.

\section{Acknowledgements}

The authors thank Dr. Stephen L. Morgan (University of South Carolina) for the provision of samples for this study, as well as Dana Bors, Wil Kranz, and Maria Diez (IUPUI) for their assistance in spectra acquisition.

Funding: Portions of this research were supported by the National Institute of Justice, Office of Justice Programs [award number 2010-DN-BX-K220] . Georgina Sauzier was supported by an Australian Postgraduate Award. 


\section{References}

1. Brandl, S.G., Criminal Investigation. 2nd ed. 2007, Upper Saddle River, NJ: Prentice Hall.

2. Houck, M.M. and J.A. Siegel, Fundamentals of Forensic Science. 2nd ed. 2010, Burlington, MA: Academic Press.

3. Robertson, J. and C. Roux, Fibers: Overview, in Encyclopedia of Forensic Sciences, J.A. Siegel and P.J. Saukko, Editors. 2013, Academic Press: Waltham. p. 109-112.

4. Apsell, P., What are Dyes? What is Dyeing?, in Dyeing Primer, J.R. Aspland, Editor. 1981, American Association of Textile Chemists and Colorists: Research Triangle Park, NC. p. 4-7.

5. Houck, M., Textiles, in Forensic Chemistry: Fundamentals and Applications, J.A. Siegel, Editor. 2016, Wiley-Blackwell: Chichester, UK. p. 40-74.

6. Palmer, R., Fibers: Identification and Comparison, in Encyclopedia of Forensic Sciences, J.A. Siegel and P.J. Saukko, Editors. 2013, Academic Press: Waltham. p. 129-137.

7. Siegel, J.A., Forensic Science: The Basics. 2007, Boca Raton, Florida: Taylor \& Francis.

8. Goodpaster, J.V. and E.A. Liszewski, Forensic analysis of dyed textile fibers. Analytical and Bioanalytical Chemistry, 2009. 394(8): p. 2009-2018.

9. Martin, P., Fibers: Color Analysis, in Encyclopedia of Forensic Sciences, J.A. Siegel and P.J. Saukko, Editors. 2013, Academic Press: Waltham. p. 148-154.

10. Eng, M., P. Martin, and C. Bhagwandin, The analysis of metameric blue fibers and their forensic significance. Journal of Forensic Sciences, 2009. 54(4): p. 841-845.

11. Eyring, M.B. and B.D. Gaudette, An Introduction to the Forensic Aspects of Textile Fiber Examination, in Forensic Science Handbook, R. Saferstein, Editor. 2005, Prentice Hall: Englewood Cliffs, NJ. p. 231-296.

12. Grieve, M.C., T. Biermann, and M. Davignon, The occurrence and individuality of orange and green cotton fibres. Science \& Justice, 2003. 43(1): p. 5-22.

13. Marcrae, R., R.J. Dudley, and K.W. Smalldon, The characterization of dyestuffs on wool fibers with special reference to microspectrophotometry. Journal of Forensic Sciences, 1979. 24(1): p. 117-129.

14. Suzuki, S., et al., Microspectrophotometric discrimination of single fibres dyed by indigo and its derivatives using ultraviolet-visible transmittance spectra. Science \& Justice, 2001. 41(2): p. 107-111.

15. Scientific Working Group on Materials Analysis, Forensic Fiber Examination Guidelines. 1999.

16. Scientific Working Group on Materials Analysis, Ultraviolet-Visible Spectroscopy of Textile Fibers. 2011.

17. National Academy of Sciences, Strengthening Forensic Science in the United States: A Path Forward. 2009, Committee on Identifying the Needs of the Forensic Sciences Community, National Research Council: Washington DC.

18. Causin, V., et al., Forensic analysis of acrylic fibers by pyrolysis-gas chromatography/mass spectrometry. Journal of Analytical and Applied Pyrolysis, 2006. 75(1): p. 43-48.

19. Gilbert, C. and S. Kokot, Discrimination of cellulosic fabrics by diffuse reflectance infrared Fourier transform spectroscopy and chemometrics. Vibrational Spectroscopy, 1995. 9(2): p. 161-167.

20. Ruckebusch, C., et al., Quantitative analysis of cotton-polyester textile blends from nearinfrared spectra. Applied Spectroscopy, 2006. 60(5): p. 539-544.

21. Daéid, N.N., W. Meier-Augenstein, and H.F. Kemp, Investigating the provenance of un-dyed spun cotton fibre using multi-isotope profiles and chemometric analysis. Rapid Communications in Mass Spectrometry, 2011. 25(13): p. 1812-1816.

22. Fortier, C. and J. Rodgers, Preliminary examinations for the identification of U.S. domestic and international cotton fibers by near-infrared spectroscopy. Fibers, 2014. 2(4): p. 264-274.

23. Liu, Y., Vibrational spectroscopic investigation of Australian cotton cellulose fibres: Part 1 - $A$ Fourier transform Raman study. Analyst, 1998. 123(4): p. 633-636. 
24. Morgan, S.L., et al. Pattern recognition methods for the classification of trace evidence textile fibers from UV/visible and fluorescence spectra. in 2007 Trace Evidence Symposium. 2007. Clearwater Beach, Florida.

25. Kramer, R., Chemometric Techniques for Quantitative Analysis. 1998, New York: Marcel Dekker, Inc.

26. Girod, A. and C. Weyermann, Lipid composition of fingermark residue and donor classification using GC/MS. Forensic Science International, 2014. 238: p. 68-82.

27. Croxton, R.S., et al., Variation in amino acid and lipid composition of latent fingerprints. Forensic Science International, 2010. 199(1-3): p. 93-102.

28. Miller, J.N. and J.C. Miller, Statistics and Chemometrics for Analytical Chemistry. 6th ed. 2010, Harlow: Prentice Hall.

29. Otto, M., Chemometrics: Statistics and computer application in analytical chemistry. 2 nd ed. 2007, Weinheim, Germany: Wiley-VCH.

30. Gemperline, P.J., Principal Component Analysis, in Practical Guide to Chemometrics. 2006, CRC Press: Boca Raton, Florida. p. 69-104.

31. Morgan, S.L. and E.G. Bartick, Discrimination of Forensic Analytical Chemical Data using Multivariate Statistics, in Forensic Analysis on the Cutting Edge: New Methods for Trace Evidence Analysis, R.D. Blackledge, Editor. 2007, John Wiley \& Sons: New Jersey. p. 333-374.

32. Varmuza, K. and P. Filzmoser, Principal Component Analysis, in Introduction to Multivariate Statistical Analysis in Chemometrics. 2009, Taylor \& Francis: Boca Raton, Florida.

33. Lavine Barry, K. and J. Workman, Chemometrics: Past, Present, and Future, in Chemometrics and Chemoinformatics. 2005, American Chemical Society. p. 1-13.

34. Kinton, V., Multivariate Techniques, in Practical Analysis of Flavor and Fragrance Materials. 2011, John Wiley \& Sons. p. 91-110.

35. Bensmail, H. and G. Celeux, Regularized gaussian discriminant analysis through eigenvalue decomposition. Journal of the American Statistical Association, 1996. 91(436): p. 1743-1748.

36. Zadora, G., Chemometrics and Statistical Considerations in Forensic Science, in Encyclopedia of Analytical Chemistry, R.A. Meyers, Editor. 2006, John Wiley \& Sons: New Jersey.

37. Mendlein, A., C. Szkudlarek, and J.V. Goodpaster, Chemometrics, in Encyclopedia of Forensic Sciences, J.A. Siegel and P.J. Saukko, Editors. 2013, Academic Press: Waltham. p. 646-651.

38. Brereton, R.G., Chemometrics: Data Analysis for the Laboratory and Chemical Plant. 2003, London: John Wiley \& Sons.

39. Scientific Working Group on Materials Analysis, Introduction to Forensic Fiber Examination. 2011.

40. Fisher, R.A., Statistical Methods for Research Workers. 13th ed. 1958, Edinburgh: Oliver and Boyd.

41. Fisher, R.A., On the interpretation of $\chi 2$ from contingency tables, and the calculation of $P$. Journal of the Royal Statistical Society, 1922. 85(1): p. 87-94.

42. Fisher, R.A., The Design of Experiments. 8th ed. 1971, New York: Hafner Publishing Company.

43. Mao, J., et al., Removal of Basic Blue 3 from aqueous solution by Corynebacterium glutamicumbiomass: Biosorption and precipitation mechanisms. Korean Journal of Chemical Engineering, 2008. 25(5): p. 1060-1064.

44. Sigma Aldrich. Basic Blue 3. 2015; Available from:

http://www.sigmaaldrich.com/catalog/product/aldrich/378011?lang=en\&region=AU.

45. Wawrzkiewicz, M., Removal of C.I. Basic Blue 3 dye by sorption onto cation exchange resin, functionalized and non-functionalized polymeric sorbents from aqueous solutions and wastewaters. Chemical Engineering Journal, 2013. 217: p. 414-425.

46. Ceron-Rivera, M., M.M. Davila-Jimenez, and M.P. Elizalde-Gonzalez, Degradation of the textile dyes Basic yellow 28 and Reactive black 5 using diamond and metal alloys electrodes. Chemosphere, 2004. 55: p. 1-10. 
47. Iranifam, M., M. Zarei, and A.R. Khataee, Decolorization of C.I. Basic Yellow 28 solution using supported ZnO nanoparticles coupled with photoelectro-Fenton process. Journal of Electroanalytical Chemistry, 2011. 659(1): p. 107-112.

48. Speers, S.J., B.H. Little, and M. Roy, Separation of acid, basic and dispersed dyes by a singlegradient elution reversed-phase high-performance liquid chromatography system. Journal of Chromatography A, 1994. 674(1-2): p. 263-70.

49. Brereton, R.G., Pattern Recognition, in Applied Chemometrics for Scientists. 2007, John Wiley \& Sons: Chichester, England. p. 145-191.

50. Adams, M.J., Chemometrics in Analytical Spectroscopy. 2nd ed. 2004, Cambridge: Royal Society of Chemistry. 